\section{J.A. Neder}

Pulmonary Function and Clinical Exercise Physiology Unit (SEFICE), Division of Respiratory Diseases, Federal University of São Paulo (UNIFESP), São Paulo, Brazil.

\section{REFERENCES}

1 Dal Corso S, Duarte SR, Neder JA, et al. A step test to assess exercise-related oxygen desaturation in interstitial lung disease. Eur Respir J 2007; 29: 330-336.

2 Palange P, Ward SA, Carlsen $\mathrm{KH}$, et al. Recommendations on the use of exercise testing in clinical practice. Eur Respir J 2007; 29: 185-209.

\title{
Tracheotomy and ventilator-associated pneumonia: the importance of oral care
}

\section{To the Editors:}

We read with interest the recent study of NsEIR et al. [1], which demonstrated that tracheotomy was independently associated with a decreased risk of ventilator-associated pneumonia (VAP). The authors offered several potential explanations as to why tracheotomised patients should be at decreased risk of VAP compared to patients with translaryngeal intubation. These included liberation of the vocal cords, resulting in a reduced risk of aspiration of contaminated oropharyngeal secretions into the lung and the reduction in bacterial biofilm formation associated with regular changing of the tracheotomy cannula, and facilitation of weaning, leading to a shorter duration of mechanical ventilation.

An additional explanation that should also be considered is differences in the quality of oral care between tracheotomised patients and those with translaryngeal intubation. There is increasing evidence that dental plaque serves as an important reservoir for respiratory pathogens implicated in VAP [2]. Indeed, some hospitals have instigated formal oral care programmes in order to reduce VAP rates in high-risk patients [3]. However, in patients intubated via the translaryngeal route, the endotracheal tube may obscure the view of the oral cavity and impede access for adequate oral care [4]. Moreover, nurses are often reluctant to administer oral care for fear of dislodging the endotracheal tube [4]. Finally, the oral tracheal tube may, by holding the mouth open, predispose to xerostomia, an important contributory factor to poor oral hygiene [5].

Since all of these problems are obviated by tracheotomy, we would postulate that improved oral care also contributed to the reduction in ventilator-associated pneumonia seen in these patients.

\section{P. Frost and M.P. Wise}

Critical Care Directorate, University Hospital of Wales, Cardiff, UK.

\section{STATEMENT OF INTEREST}

None declared.

\section{REFERENCES}

1 Nseir S, Di Pompeo C, Jozefowicz E, et al. Relationship between tracheotomy and ventilator-associated pneumonia: a case-control study. Eur Respir J 2007; 30: 314-320.

2 El-Solh AA, Pietrantoni C, Bhat A, et al. Colonization of dental plaques: a reservoir of respiratory pathogens for hospital acquired pneumonia in institutionalized elders. Chest 2004; 126: 1575-1582.

3 Schleder B, Stott K, Lloyd RC. The effect of a comprehensive oral care protocol on patients at risk for ventilator associated pneumonia. J Advocate Health Care 2002; 4: 27-30.

4 McNeill HE. Biting back at poor oral hygiene. Intensive Crit Care Nurs 2000; 16: 367-372.

5 Munro CL, Grap MJ. Oral health and care in the intensive care unit: state of the science. Am J Crit Care 2004; 13: 25-33.

DOI: $10.1183 / 09031936.00107207$

From the authors:

We would like to thank P. Frost and M.P. Wise for their comments, and wish to respond to some of the points they raised.

Dental plaque, the oropharyngeal cavity and the stomach are potential reservoirs for microorganisms in critically ill patients. Aspiration of contaminated oropharyngeal and gastric secretions is common in intensive care unit (ICU) patients requiring mechanical ventilation through an endotracheal tube or a tracheotomy cannula [1]. The most important mechanism of ventilator-associated pneumonia (VAP) is gross or microaspiration of oropharyngeal microorganisms into the distal bronchi, followed by bacterial proliferation and parenchymal invasion, leading to bronchopneumonia [2]. Aspiration of oropharyngeal contents containing a large bacterial inoculum overwhelms host defences that are already compromised by critical illness and the presence of an endotracheal tube, thus leading to the development of VAP. Understanding this sequence of pathophysiological events, it would seem logical that reducing concentrations of oral microorganisms would have a beneficial effect on prevention of VAP [3]. 